\title{
Self-Compassion bei Kindern und Jugendlichen
}

\author{
Armin Castello \\ Europa-Universität Flensburg
}

Dass Kinder und Jugendliche familiäre, gesundheitliche oder entwicklungsbedingte Beeinträchtigungen oder soziale Stigmatisierung erleben, ist eine zentrale pädagogische Herausforderung. Eine Veränderung der Lebenssituation Betroffener kann durch die Reduktion vorhandener Risikofaktoren und die Stärkung von Kompetenzen zu deren Bewältigung erreicht werden. Das Konstrukt der Self-Compassion, abgeleitet aus der buddhistischen Psychologie, bietet in diesem Zusammenhang neue Anknüpfungspunkte, deren Relevanz sich zunehmend in der pädagogisch-psychologischen Befundlage widerspiegelt, die hier zusammenfassend dargestellt wird.

\section{Self-Compassion: Mitgefühl für einen selbst}

Self-Compassion besteht in einer Haltung, die als Fürsorge und Freundlichkeit gegenüber der eigenen Person beschrieben werden kann. Diese wird auch dann aufrechterhalten, wenn Ereignisse eintreten, die einen selbst in einem negativen Licht erscheinen lassen (Neff, 2003 b). Self-Compassion zeigt sich, wenn man sich in belastenden Situationen mit Verständnis und Mitgefühl gegenübertritt, sich weder verurteilt noch harsch kritisiert oder beschämt (Karl et al., 2018). Self-Compassion wirkt insofern auch als eine adaptive Strategie zur Emotionsregulation, durch die in Belastungssituationen positive, an das Selbst gerichtete Emotionen ermöglicht werden (Berking und Whitley, 2014).
Neff (2003 a) beschreibt Self-Compassion in drei Kerndimensionen als

praktizierte Freundlichkeit statt einer Selbstverurteilung,

- eine empfundene Zugehörigkeit zur Gemeinschaft statt erlebter Isolation und

v praktizierte Achtsamkeit anstatt einer unangemessenen Identifizierung mit negativen Ereignissen.

Auf der Basis dieser Konzeption wurde ein Instrument entwickelt - die Self-Compassion Scale (SCS) (ebd.) - mit dessen Hilfe eine Operationalisierung des Konstrukts erfolgen soll.

\section{Entwicklung von Self-Compassion}

In der individuellen Entwicklung von SelfCompassion spielen zumeist frühkindliche Erfahrungen eines unbedingten geliebt Seins eine grundlegende Rolle (Gilbert, 2009). Das Internalisieren von positiven Interaktionserfahrungen durch die elterliche Sensitivität und Responsivität (de Wolff \& van Ijzendoorn, 1997) erleichtert, dass Kinder oder Jugendliche sich selbst gegenüber bei negativen emotionalen Zuständen Freundlichkeit und Fürsorge zeigen können. Ein unsicherer Bindungsstil ist häufiger verbunden mit Selbstkritik und weniger positiven selbstgerichteten Emotionen bei negativen Erfahrungen (Kopala-Sibley et al., 2013). 
Restriktives und überkontrollierendes Elternverhalten, Ablehnung oder persistierende Kritik und ein Mangel an emotionaler Wärme wurden als Faktoren identifiziert, die der Entwicklung von Self-Compassion entgegenstehen (Campos, Besser \& Blatt, 2010). Insbesondere sich wiederholende, beschämende Ereignisse können dramatisch negative Auswirkungen auf die Entwicklung des Selbstkonzepts haben (Steindl, Matos \& Creed, 2018). Häufen sich blamierende Rückmeldungen zur eigenen Person durch die soziale Umwelt auch in pädagogischen Einrichtungen durch Gleichaltrige oder erwachsene Personen können Kinder eine zunehmend negative Sicht auf sich selbst entwickeln und andere als bedrohlich und urteilend erleben.

\section{Wirkrichtungen und Einfluss von Self-Compassion}

Soziale Ausgrenzung und Stigmatisierung werden von Kindern und Jugendlichen in aller Regel als erhebliche psychische Belastung erlebt. Self-Compassion ist ein wirksamer Schutzfaktor, wenn Menschen mit Erfahrungen konfrontiert sind, die als belastend, beschämend oder als Beeinträchtigung ihres Selbstkonzepts wirken können (Barnard \& Curry, 2011). Selbstmitgefühl unterstützt Schülerinnen und Schüler in Situationen einer erlebten Ausgrenzung, da sie auch bei einer fundamentalen Infragestellung der eigenen Person selbstgerichtet Wärme, Akzeptanz und Verbundenheit empfinden können (Vigna, Poehlmann-Tynan \& Koenig, 2018).

Menschen, die sich selbst gegenüber Mitgefühl zeigen, entwickeln ein insgesamt größeres emotionales Wohlbefinden (Bluth et al., 2016). Die Metaanalyse von MacBeth und Gumley (2012) untersuchte in insgesamt 20 Studien den Zusammenhang zwischen Self-Compassion einerseits und internalisierenden Störungen wie Angst und Depression andererseits.
Dabei zeigten sich mehrheitlich hohe (inverse) Effektstärken. Menschen, die im Kontext einer Depression mit Self-Compassion statt mit Selbstkritik auf negative emotionale $\mathrm{Zu}$ stände reagieren, d.h. Akzeptanz statt Vermeidung zeigen, reduzieren auf diese Weise die negativen emotionalen Zustände (Krieger, Berger \& Holtforth, 2016). Self-Compassion zeigt sich zudem als hilfreich, um die Auswirkungen von exzessivem Perfektionismus zu kompensieren.

Self-Compassion scheint unmittelbar mit positiven psychologischen Zuständen wie Glück, Optimismus und Lebenszufriedenheit verbunden zu sein (Hollis-Walker \& Colosimo, 2011). Selbstgerichtetes Mitgefühl stärkt außerdem günstiges Verhalten in Bezug auf die eigene Gesundheit und eine positivere Körperwahrnehmung (Albertson, Neff \& Dill-Shackleford, 2014). Es ist verknüpft mit empathischem Verhalten anderen gegenüber, größerer Bereitschaft und Fähigkeit zum Perspektivenwechsel und dahingehend, anderen zu verzeihen (Neff \& Pommier, 2013).

Dass Self-Compassion sich positiv auf das Kommunikationsverhalten auswirkt, konnten Long und Neff (2018) belegen. Die individuelle Tendenz, sich bspw. in Gruppeninteraktionen zu beteiligen, die Bereitschaft, Fragen zu stellen, Hilfe zu suchen und diese anzunehmen und sich mit anderen auszutauschen, wird erleichtert, da sich die Angst vor Fremdbewertung reduziert.

\section{Unterstützung der Entwicklung von Self-Compassion}

Mit den individuellen biografischen Erfahrungen entstehen große Unterschiede bezüglich Self-Compassion. Durch pädagogische und therapeutische Interventionen kann eine Stärkung erreicht werden, die mit einer Reihe positiver Effekte verbunden ist (Gilbert, 2009). 
So legten Smeets et al. (2014) ein dreiwöchiges Interventionsprogramm für Schülerinnen und Schüler an weiterführenden Schulen vor. Bestandteile dieses Programms waren eine Kombination aus Diskussionen von erlebten Belastungen, körperbezogenen Übungen und das Fokussieren einer „inneren kritischen Stimme“, der gezielt selbstgerichtetes Mitgefühl entgegengestellt wurde.

Das Verfassen eines mitfühlenden Briefs, den Schülerinnen und Schüler an sich selbst richten, untersuchten Shapira und Mongrain (2010). Sie hatten die Aufgabe, sich dort selbstgerichtet bezüglich eines belastenden Ereignisses in einer verständnisvollen Weise zu äußern, ähnlich wie gegenüber einem guten Freund. Nach einer Interventionswoche wurde ein Rückgang depressiver Symptome und, langfristig, positive Effekte hinsichtlich des allgemeinen Wohlbefindens erkennbar.

Tatsächlich scheinen bereits einfache Übungen zur Aktivierung von Self-Compassion positive Effekte zu haben (Arimitsu \& Hofmann, 2017). Dundas et al. (2017, S. 448) beschrieben die Möglichkeiten, wie Selbstmitgefühl im Schulkontext durch verschiedene Aktivitäten unterstützt werden kann, wie z. B.

- sich wie einem Freund/einer Freundin gegenüber zu verhalten, wenn einem etwas an einem selbst nicht gefällt,

- ein freundliches Reagieren auf Belastungsreaktionen des eigenen Körpers,

- das explizite Thematisieren von schulischer Beschämung und deren Auflösung sowie

- das Benennen und Relativieren von Selbstkritik bei erlebtem schulischem „Versagen“.

Auch eine einfache Beschäftigung mit dem Thema Selbstmitgefühl scheint positive Effekte $\mathrm{zu}$ entwickeln (Neff, Kirkpatrick \& Rude, 2007). Es bewirkt, dass Menschen schmerzvolle Erkenntnisse nicht vermeiden, sondern diese aushalten und sich selbst gegenüber freund- liche Aufmerksamkeit entwickeln. In der pädagogischen Unterstützung selbstgerichteten Mitgefühls liegt insofern eine durchaus sehr vielversprechende pädagogische Perspektive.

\section{Literatur}

Albertson, E.R., Neff, K.D. \& Dill-Shackleford, K.E. (2014). Self-compassion and body dissatisfaction in women: A randomized controlled trial of a brief meditation intervention. Mindfulness, 6 (3) 1-11. https://doi.org/10.1007/s126 71-014-0277-3

Arimitsu, K. \& Hofmann, S.G. (2017). Effects of compassionate thinking on negative emotions. Cognition and Emotion, 31 (1), 160-167. https://doi.org/10.1080/02699931.2015.1078292

Barnard, L. K. \& Curry, J.F. (2011). Self-compassion: conceptualizations, correlates, and interventions. Review of General Psychology, 15 (4), 289-303. https://doi.org/10.1037/a0025754

Berking, M. \& Whitley, B. (2014). Affect Regulation Training (ART). New York, NY: Springer.

Bluth, K., Roberson, P. N., Gaylord, S.A., Faurot, K. R., Grewen, K.M., Arzon, S. \& Girdler, S. S. (2015) Does self-compassion protect adolescents from stress? Journal of Child and Family Studies, 25 (4), 1098-1109. https://doi.org/10.1037/ aoo25754

Campos, R.C., Besser, A. \& Blatt S.J. (2010). The mediating role of selfcriticism and dependency in the association between perceptions of maternal caring and depressive symptoms. Depression and Anxiety, 27 (12), 1149-1157. https://doi.org/10.1037/aoo25754

De Wolff, M. S. \& van ljzendoorn, M. H. (1997). Sensitivity and attachment: A meta-analysis on parental antecedents of infant attachment. Child Development, 68 (4), 571-591. https:/l doi.org/10.2307/1132107

Dundas, I., Binder, P.-E., Hansen, T. G. B. \& Stige, S. H. (2017). Does a short self-compassion intervention for students increase healthy self-regulation? A randomized control trial. Scandinavian Journal of Psychology, 58(5), 443-450. https:/l doi.org/10.1111/sjop.12385

Gilbert, P. (2009). The Compassionate Mind: A New Approach to Life's Challenges. London: Constable \& Robinson. 
Hollis-Walker, L. H. \& Colosimo, K. (2011). Mindfulness, self-compassion, and happiness in nonmeditators: A theoretical and empirical examination. Personality and Individual Differences, 50 (2), 222-227. https://doi.org/10.1016/j.paid. 2010.09 .033

Karl, A., Williams, M. J., Cardy, J., Kuyken, W. \& Crane, C. (2018). Dispositional self-compassion and responses to mood challenge in people at risk for depressive relapse/recurrence. Clinical Psychology and Psychotherapy, 25 (5), 621-633. https://doi.org/10.1002/cpp.2302

Kopala-Sibley, D.C., Zuroff, D.C., Leybman, M. J. \& Hope, N. (2013). Recalled peer relationship experiences and current levels of selfcriticism and self-reassurance. Psychological Psychotherapy, 86 (1), 33-51. https://doi.org/10.1111/ j.2044-8341.2011.02044.x

Krieger, T., Berger, T. \& Holtforth, M. G. (2016). The relationship of self-compassion and depression: Cross-lagged panel analyses in depressed patients after outpatient therapy. Journal of Affective Disorders, (202), 39-45. https:/ldoi. org/10.1016/j.jad.2016.05.032

Long, P. \& Neff, K. D. (2018). Self-compassion is associated with reduced self-presentation concerns and increased student communication behavior. Learning and Individual Differences, 67, 223-231. https://doi.org/10.1016/j.lin dif.2018.09.003

MacBeth, A. \& Gumley, A. (2012) Exploring compassion: a meta-analysis of the association between self-compassion and psychopathology. Clinical Psychology Review, 32 (6). 545-552. https://doi.org/10.1016/j.cpr.2012.06.003

Neff, K. D. (2003 a). Development and validation of a scale to measure self-compassion. Self and Identity, 2, 223-250.

Neff, K.D. (2003 b). Self-compassion: An alternative conceptualization of a healthy attitude toward oneself. Self and Identity, 2 (2), 85-102. https://doi.org/10.1080/15298860309032

Neff, K.D. (2009). The role of self-compassion in development: A healthier way to relate to oneself. Human Development, 52 (4), 211-214. https://doi.org/10.1159/000215071
Neff, K.D. \& Pommier, E. (2013). The relationship between self-compassion and other-focused concern among college undergraduates, community adults, and practicing meditators. Self and Identity, 12 (2), 160-176. https://doi.org/ 10.1080/15298868.2011.649546

Neff, K. D., Kirkpatrick, K. L. \& Rude, S. S. (2007). Self-compassion and adaptive psychological functioning. Journal of Research in Personality, 41 (1), 139-154. https://doi.org/10.1016/j. jrp.2006.03.004

Shapira, L. B., \& Mongrain, M. (2010). The benefits of self-compassion and optimism exercises for individuals vulnerable to depression. The Journal of Positive Psychology, 5 (5), 377-389. https://doi.org/10.1080/17439760.2010.516763

Smeets, E., Neff, K., Alberts, H. \& Peters, M. (2014). Meeting suffering with kindness: Effects of a brief self-compassion intervention for female college students. Journal of Clinical Psychology, 70 (9), 794-807. https://doi.org/10.1002/ jclp.22076

Steindl, S.R., Matos, M. \& Creed, A.K. (2018). Early shame and safeness memories, and later depressive symptoms and safe affect: the mediating role of self-compassion. Current Psychology, 1-11. https://doi.org/10.1007/s1 21 44-018-9990-8

Vigna, A.J., Poehlmann-Tynan, J. \& Koenig, B.W. (2018). Does self-compassion facilitate resilience to stigma? A school-based study of sexual and gender minority youth. Mindfulness, 9 , 914-924. https://doi.org/10.1007/s12671-017o831-x

\section{Anschrift des Autors}

\author{
Prof. Dr. Armin Castello
}

Europa-Universität Flensburg

Institut für Sonderpädagogik

Auf dem Campus 1

D-24943 Flensburg

E-Mail: armin.castello@uni-flensburg.de 